\title{
Production of L-Arginine from arginine analogue resistant mutants of Corynebacterium glutamicum
}

\author{
Anjaneyulu Musini ${ }^{1}$, Lakshmi Narasu Mangamoori ${ }^{2}$, L. Saida $^{3} *$ \\ ${ }^{1}$ Centre for Biotechnology, Institute of Science \& Technology, Jawaharlal Nehru Technological University, Hyderabad, INDIA \\ ${ }^{2}$ Centre for Biotechnology, Institute of Science \& Technology, Jawaharlal Nehru Technological University, Hyderabad, INDIA \\ ${ }^{3}$ Centre for Biotechnology, Institute of Science \& Technology, Jawaharlal Nehru Technological University, Hyderabad, INDIA \\ *Corresponding Author: lavudisaida@jntuh.ac.in
}

Available online at: www.isroset.org

Received: 16/Sept/2018, Accepted: 28/Sept/2018, Online: 31/Oct/2018

\begin{abstract}
L-arginine is an essential amino acid, widely used in pharmaceutical, feed and food industry. It is the precursor molecule in citrulline and nitric oxide synthesis, which in turn plays significant role in a wide variety of patho - physiological functions in the cardiovascular, central and peripheral nervous systems. In the present study, mutagenesis experiments were carried out for isolation of analogue resistant mutants against Corynebacterium glutamicum. Initially, MIC (Minimum inhibitory concentration) was identified for arginine analogues by tube dilution method. MIC values found to be Arginine hydroximate $-680 \mu \mathrm{g} / \mathrm{ml}$. Further, mutagenesis of C. glutamicum was carried out with NTG $(300 \mu \mathrm{g} / \mathrm{ml})$ and amino acid analogue resistant mutants were isolated in minimal media. Fermentation was carried out by resistant colonies and estimated the Arginine levels. Production level was found to be $5.2 \mathrm{mg} / \mathrm{ml}$ where as in control cells it was $1.4 \mathrm{mg} / \mathrm{ml}$. This study highlighted the $C$. glutamicum mutant of L-arginine amino acid production.
\end{abstract}

Key words: L-Arginine, Mutagenesis, Minimum inhibitory concentration and Corynebacterium glutamicum

\section{INTRODUCTION}

The production of L-arginine is generally carried out by chemical synthesis or extraction of protein hydrolysates from L-ornithine produced by the process of fermentation. So far, a highly desirable method, the direct fermentation of $\mathrm{L}$ - arginine, is not available.

There are eight steps in the arginine biosynthesis pathway from glutamate, the first precursor of cycle. This pathway is regulated by the levels of arginine present in a wide variety of bacteria [1, 2, 3, 4, 5]. Arginine-requiring mutants produce large amounts of citruline or ornithine or when arginine is limiting $[6,7]$ although their wild-type strains donot accumulate arginine due to these feedback controls by arginine. Arginine produced by mutants with a defective regulatory mechanism and that regulatory mutants could be found among the group of analogue- resistant mutants.

L-arginine biosynthesis was regulated by two regulatory patterns in different microorganisms [8]. Corynebacterium glutamicum, a glutamic acid producing bacteria, has advantage as an L-arginine producer because $\mathrm{N}$ acetylglutamokinase, enzyme in the biosynthesis is subjected to feedback inhibition by L-arginine and also it carries strong flux towards L-glutmate formation. Importantly, for the high yield of L-arginine, the repression of regulator gene (encoded by $\operatorname{argR}$ ) in the operon coding L-arginine biosynthesis ( $\arg$ ) is considered as one parameter as well the feedback inhibition of $\mathrm{N}$-acetylglutamate kinase as second parameter $[9,10]$.

In the present study, glutamic acid producing bacteria, $C$. glutamicum was examined after treating with mutagen in the presence of amino acid analogue on minimal media. The amino acid production was found to be more in resistant mutant compared to wild strain.

\section{MATERIAL AND METHODS}

\subsection{Bacteria culture:}

Corynebacterium glutamicum strain was procured form MTCC, IMTEC, Chandigarh India. The culture was maintained on Mueller hinton agar slant at $4^{\circ} \mathrm{C}$, revived in Mueller hinton broth and cultured at $37^{\circ} \mathrm{C}$ before their use.

\subsection{Mutagenesis:}

A loop full of the active culture was inoculated into the nutrient broth and incubated. $50 \mu \mathrm{l}$ of the above grown 
culture was added to each of the six sterile boiling tubes that contain $5 \mathrm{ml}$ nutrient broth and incubated for $8-10$ hours (until the O.D reaches 0.8 ) in a shaking incubator at $37^{\circ} \mathrm{C}$ and $200 \mathrm{rpm}$. Centrifuge the samples at $8000 \mathrm{rpm}$ for 15 minutes. The cell pellet was dissolved in $5 \mathrm{ml}$ citrate buffer $(\mathrm{pH}-5.5)$. Concentration of NTG used was $200-600 \mu \mathrm{g} / \mathrm{ml}$. Incubate the tubes at $37^{\circ} \mathrm{C}$ in an incubator for $45 \mathrm{~min}$. Collect the cell pellet and wash twice by using minimal $\mathrm{A}$ buffer $\left(\mathrm{K}_{2} \mathrm{HPO}_{4}, \mathrm{KH}_{2} \mathrm{PO}_{4},\left(\mathrm{NH}_{4}\right)_{2} \mathrm{PO}_{4}\right.$ and Sodium Citrate $)$ solution. Finally the cell pellet was dissolved in $2 \mathrm{ml}$ minimal buffer solution. $0.1 \mathrm{ml}$ of each microbial suspension was serially diluted up to $10^{-4}$ dilutions. $0.1 \mathrm{ml}$ of the above microbial suspension from each of the $10^{-4}$ dilution tube was spread plated onto the respective minimal media plate. The plates were incubated at $37^{\circ} \mathrm{C}$ for 24 hours. Numbers of colonies on each plate were counted and compared with that of the control plate. The concentration at which the number of colonies would be reduced to $50 \%$, when compared to number of colonies on the control plate will be considered as optimized concentration. [11]

\subsection{Fermentation:}

Seed media: Glucose $-2 \%$, Peptone $-1 \%$, Yeast Extract $1 \%, \mathrm{Nacl}-0.5 \%$, initial $\mathrm{p}^{\mathrm{H}}-6.52$, Adjusted $\mathrm{P}^{\mathrm{H}}-7.2$ (Before sterilization). $2 \%$ glucose was added to seed media, seperately after sterilization. A loop full of cultures inoculated in to seed media. Incubation kept at $30^{\circ} \mathrm{C}, 200$ rpm for overnight.

Production media: $\left(\mathrm{NH}_{4}\right)_{2} \mathrm{SO}_{4}-3 \%, \mathrm{KH}_{2} \mathrm{PO}-0.05 \%$, $\mathrm{K}_{2} \mathrm{HPO}_{4}-0.05 \%, \mathrm{MgSO}_{4}-0.025 \%, \mathrm{CaCO}_{3}-2 \%$, Initial pH6.84, Adjusted $\mathrm{P}^{\mathrm{H}}-7.2$ (Before sterilization). Glucose was sterilized separately, after cooling the media $10 \%$ glucose was added to production media. $4 \%$ inoculums of culture inoculated to production media. Incubation kept at $30^{\circ} \mathrm{C}, 200$ rpm [12].

\subsection{Estimation of arginine by Sakaguchi test:}

Inoculated the isolated resistant colony into seed media and then into production media. Estimation of L-Arginine through Sakaguchi test was done for every 24 hours [13]. Arginine standard solution was prepared by dissolving $0.1 \mathrm{gm}$ arginine in $10 \mathrm{ml}$ of sterile water. Different concentration range 4 - $36 \mu \mathrm{g}$ was used for construction of standard graph. Double distilled water was added to make up the final volume to $4 \mathrm{ml}$ to which $1 \mathrm{ml}$ of $\mathrm{KOH}$ to each tube was added and mix gently. $2 \mathrm{ml}$ of $0.1 \%$ naphthalene was added and then $1 \mathrm{ml}$ of $40 \%$ urea was added and mixed gently. Tubes were kept at $4^{0} \mathrm{C}$ for $10 \mathrm{~min}$. $1 \mathrm{ml}$ Potassium hypobromite was added and gently mixed. O.D. values were taken by UV Spectrophotometer at 540nm. Standard Graph was constructed and concentration of Arginine in culture was checked at different time points.

\section{RESULTS AND DISCUSSION}

\subsection{Mutagenesis:}

Amino acid analogue arginine hyroxamate inhibited the growth by different bacteria and also reversed in the presence of arginine amino acid [14]. In order to isolate arginine analogue resistant mutant initially, the minimum inhibitory concentration of arginine hydroxamate was determined by tube dilution method. MIC of the arginine hydroxamate was found to be $683 \mu \mathrm{g} / \mathrm{ml}$ against $C$. glutamicum and same concentration was used for isolation of amino acid analogue resistant mutant. Strain improvements play an essential role in the commercial development of microbial fermentation [15]. In the recent years, new methods such as rationale screening and recombinant DNA technology have begun to make a significant contribution to this activity [16]. However, mutagenesis and random screening is still cost effective process and reliable short-term strain development is frequently the method of choice.

Chemical mutagenesis by methylnitronotrosoguanidine (MNG or NTG) is a successful method to the strain development for high yield production [17]. In the present study, NTG was used for mutagenesis experiment. The methylnitronotrosoguanidine (MNNG or $\mathrm{MNG}$ ) mutagen concentration was optimized towards Corynebacterium glutamicum. Culture was treated with different MNG concentrations and calculated the survival rate of the organism. Graph of mutagenesis concentration verses survival rate of bacteria shown in Fig $1.50 \%$ growth was inhibited at $300 \mu \mathrm{g} / \mathrm{ml}$ MNG concentration, similar concentration used for the mutagenesis experiment for isolation of resistant colonies. The concentration at which the number of colonies would be reduced to $50 \%$, when compared to number of colonies on the control plate will be considered as optimized concentration.

The concentration at which the number of colonies would be reduced to $50 \%$, when compared to number of colonies on the control plate will be considered as optimized concentration. After treating with optimized concentration of MNG, culture was grown on minimal media containing arginine hydroxamate $(893 \mu \mathrm{g} / \mathrm{ml})$ and after incubation of few days three resistant mutant colonies were obtained shown on Figure 2.

After obtaining resistant mutants, colonies were grown on same analogue concentration in the minimal media for stabilization of mutants.

\subsection{Fermentation and estimation of arginine}

Fermentation was carried out at shake flask level by mutant colonies and wild strain. Culture was incubated over night in to the seed media and next transfers to the production media. 
Seed media and production media composition given in materials and method section. Every $24 \mathrm{~h}$ interval culture sample was collected and arginine amino acid was estimated. Sakaguchi spectrophotometer method was used for analysis. Under alkaline condition $\alpha$ - naphthol react with guanidine group of arginine amino acid, which upon treatment with hypo bromate, produces characteristic red color. Arginine amino acid production from different resistant mutants and control wild strain was shown in figure 3.

After mutagenesis three colonies Cgarghx1, Cgarghx5 and Cgarghx10 were obtained and these three colonies are purified and check for arginine production. Among all purified colonies Cgarghx 1 was produced more amino acid production $(5.2 \mathrm{mg} / \mathrm{ml})$ at $72 \mathrm{~h}$ compared to the other mutant and in the wild strain only $1.4 \mathrm{mg} / \mathrm{ml}$ arginine amino acid production. In the resistant mutant strain amino acid production was more due to regulatory abnormalities of the arginine biosynthetic enzymes [12].

\section{CONCLUSION}

Literature has shown not many reports on L-Arginine amino acid production from Cornybacteriaum glutamicum till date. In the present study, amino acid analogue resistant mutant for production of L-arginine was isolated by classical mutagenesis. Amino acid production was observed to be 5.2 $\mathrm{mg} / \mathrm{ml}$ at shake flask level fermentation. However, the future strategy would include the optimization of media components for higher yield.

\section{ACKNOWLEDGEMENT}

We are thankful the DST-TIFAC for financial support to carried out this work. We also thanks to Chidepudi Devi for manuscript preparation.

\section{CONFLICT OF INTEREST}

Author has no conflict of interest.

\section{AUTHORS' CONTRIBUTIONS}

MLN has given the concept and designed the experiments, LS has monitored the experimental work, AM has carried out the experiments.

\section{REFERENCE}

[1]. Gorini, Luigi, Wenche Gundersen, and Mikulas Burger. "Genetics of regulation of enzyme synthesis in the arginine biosynthetic pathway of Escherichia coli." In Cold Spring Harbor symposia on quantitative biology, vol. 26, pp. 173182. 1961.
[2]. Maas, Werner K. "Studies on repression of arginine biosynthesis in Escherichia coli." In Cold Spring Harbor symposia on quantitative biology, vol. 26, pp. 183-191., 1961.

[3]. Udaka, Shigezo. "Pathway-specific pattern of control of arginine biosynthesis in bacteria." Journal of bacteriology 91 , no. 2 : 617-621, 1966.

[4]. Vogel, Henry J. "Aspects of repression in the regulation of enzyme synthesis: pathway-wide control and enzyme-specific response." In Cold Spring Harbor symposia on quantitative biology, vol. 26, pp. 163-172., 1961.

[5]. Vyas, S., and W. K. Maas. "Feedback inhibition of acetylglutamate synthetase by arginine in Escherichia coli." Archives of biochemistry and biophysics 100, no. 3: 542-546, 1963.

[6]. Kinoshita, S., K. Nakayama, and S. Udaka.. The fermentative production of L-ornithine. Journal of General and Applied Microbiology, 3:276-277, 1957.

[7]. Okumura, Shinji, Masao Shibuya, Shimpachi Konishi, Moriyoshi Ishida, and Teruo Shiro. "The fermentative production of L-citrulline." Agricultural and Biological Chemistry 28, no. 10: 742-743, 1964.

[8]. S. Udaka, Amino Acids Nucleic Acids, Vol. 1, No. 14, 1966.

[9]. Ikeda, Masato, Satoshi Mitsuhashi, Kenji Tanaka, and Mikiro Hayashi. "Reengineering of a Corynebacterium glutamicum L-arginine and L-citrulline producer." Applied and environmental microbiology 75, no. 6: 1635-1641, 2009.

[10].Xu, Meijuan, Zhiming Rao, Juan Yang, Haifeng Xia, Wenfang Dou, Jian Jin, and Zhenghong $\mathrm{Xu}$. "Heterologous and homologous expression of the arginine biosynthetic $\operatorname{argC} \sim \mathrm{H}$ cluster from Corynebacteriumcrenatum for improvement of 1arginine production." Journal of industrial microbiology \& biotechnology 39, no. 3: 495-502, 2012.

[11].Yu, Guanghai, Xiaoqiang Jia, Jianping Wen, Wenyu Lu, Guoying Wang, Qinggele Caiyin, and Yunlin Chen. "Strain Improvement of Streptomyces roseosporus for daptomycin production by rational screening of $\mathrm{He}-\mathrm{Ne}$ laser and NTG induced mutants and kinetic modeling." Applied biochemistry and biotechnology 163, no. 6: 729-743, 2011.

[12].Hagino, Hiroshi, and Kiyoshi Nakayama. "L-Tyrosine production by analog-resistant mutants derived from a phenylalanine auxotroph of Corynebacterium glutamicum." Agricultural and Biological Chemistry 37, no. 9: 2013-2023, 1973.

[13].Chatterjea, M. N. Textbook of biochemistry for dental/nursing/pharmacy students. JAYPEE BROTHERS PUBLISHERS, 2004.

[14].Kisumi, Masahiko, Jyoji Kato, Masaki Sugiura, and Ichiro Chibata. "Production of L-arginine by arginine hydroxamateresistant mutants of Bacillus subtilis." Applied microbiology 22, no. 6: 987-991, 1971.

[15]. Rowlands, R. T. "Industrial strain improvement: mutagenesis and random screening procedures." Enzyme and microbial technology 6, no. 1: 3-10, 1984. 
[16].Olano, Carlos, Felipe Lombo, Carmen Mendez, and Jose A. Salas. "Improving production of bioactive secondary metabolites in actinomycetes by metabolic engineering." Metabolic engineering 10, no. 5: 281-292, 2008.

[17].Kumar, Kuttanpillai Santhosh, Ayyachamy Manimaran, Kugen Permaul, and Suren Singh. "Production of $\beta$-xylanase by a Thermomyces lanuginosus MC 134 mutant on corn cobs and its application in biobleaching of bagasse pulp." Journal of bioscience and bioengineering 107, no. 5: 494-498, 2009.

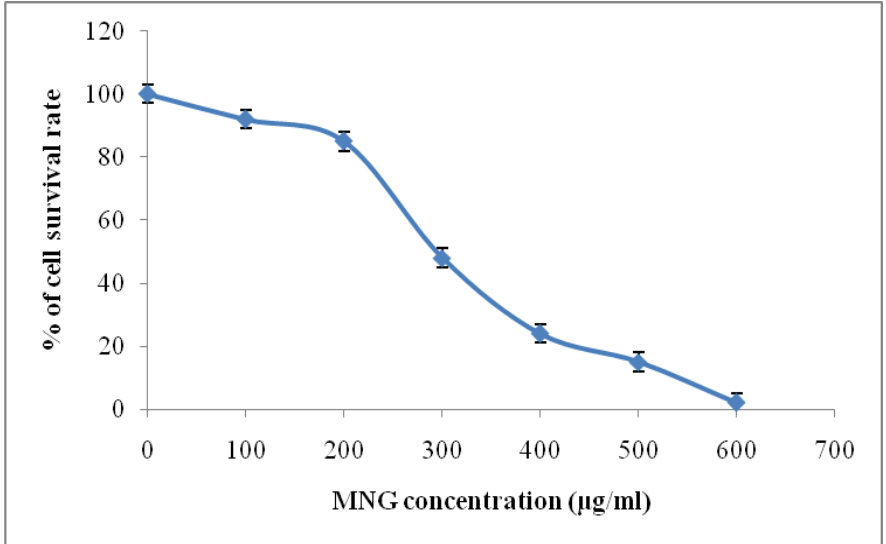

Figure 1: Survival rate of C. glutamicumwith MNG at different concentrations

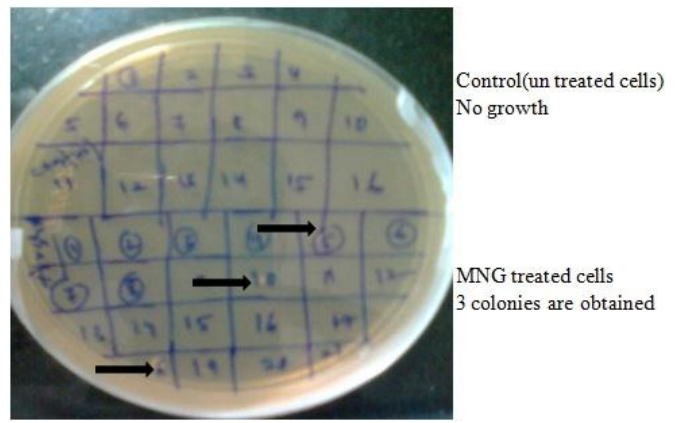

Figure 2: Isolation of Arginine hydroxamate $(983 \mu \mathrm{g} / \mathrm{ml})$ resistant colonies of Corynebacterium glutamicum by classical mutagenesis

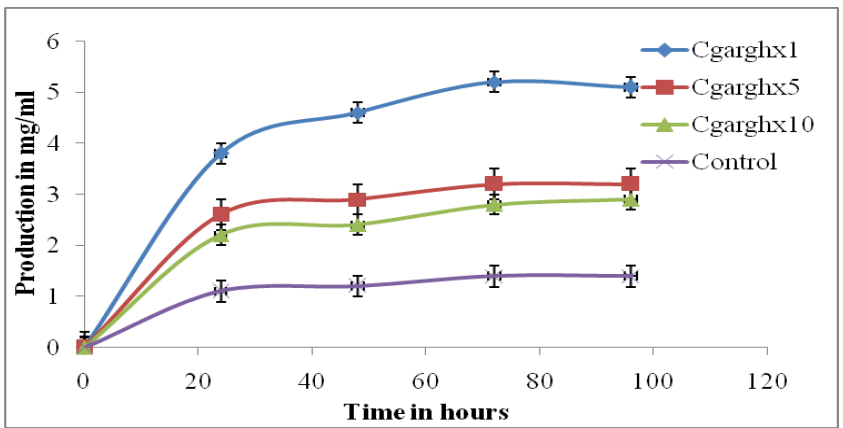

Figure 3: Arginine amino acid production from arginine hydroxamate resistant mutants and wild strain estimated by sakaguchi test 